\title{
BMJ Open Cohort profile: follow-up of a Berlin Aging Study II (BASE-II) subsample as part of the GendAge study
}

\author{
Ilja Demuth (1D ,1,2 Verena Banszerus, ${ }^{1}$ Johanna Drewelies, ${ }^{3}$ Sandra Düzel, ${ }^{4}$ \\ Ute Seeland, ${ }^{5,6}$ Dominik Spira, ${ }^{1}$ Esther Tse, ${ }^{7}$ Julian Braun, ${ }^{8,9}$ \\ Elisabeth Steinhagen-Thiessen, ${ }^{1}$ Lars Bertram, ${ }^{10,11}$ Andreas Thiel, ${ }^{8,9}$ \\ Ulman Lindenberger, ${ }^{4,12}$ Vera Regitz-Zagrosek, ${ }^{6,7,13}$ Denis Gerstorf, ${ }^{3}$ Additional \\ BASE-II/GendAge investigators
}

To cite: Demuth I, Banszerus V, Drewelies J, et al. Cohort profile: follow-up of a Berlin Aging Study II (BASE-II) subsample as part of the GendAge study. BMJ Open 2021;11:e045576. doi:10.1136/ bmjopen-2020-045576

- Prepublication history for this paper is available online. To view these files, please visit the journal online (http://dx.doi. org/10.1136/bmjopen-2020045576).

Received 05 0ctober 2020 Accepted 20 May 2021

Check for updates

(c) Author(s) (or their employer(s)) 2021. Re-use permitted under CC BY-NC. No commercial re-use. See rights and permissions. Published by BMJ.

For numbered affiliations see end of article.

Correspondence to Professor Ilja Demuth; ilja.demuth@charite.de

\section{ABSTRACT}

Purpose The study 'Sex- and gender-sensitive prevention of cardiovascular and metabolic disease in older adults in Germany', the GendAge study, focuses on major risk factors for cardiovascular and metabolic diseases and on the development of major outcomes from intermediate phenotypes in the context of sex and gender differences. It is based on a follow-up examination of a subsample (older group) of the Berlin Aging Study II (BASE-II).

Participants The GendAge study assessments took place between 22 June 2018 and 10 March 2020. A total of 1100 participants (older BASE-II subsample, aged $\geq 65$ years) with baseline data assessed at least by one of the BASE-II partner sites were investigated in the follow-up. These participants had a mean age of 75.6 years (SD \pm 3.8 ), with a mean follow-up at 7.4 years ( $S D \pm 1.5$ ). Findings to date Data from different domains such as internal medicine, geriatrics, immunology and psychology were collected, with a focus on cardiometabolic diseases and in the context of sex and gender differences. Diabetes mellitus type 2 was reported by $15.6 \%$ and $8.6 \%$ of men and women, respectively. In contrast, this disease was diagnosed in $20.7 \%$ of men and $13.3 \%$ of women, indicating that a substantial proportion of almost $30 \%$ was unaware of the disease. Echocardiography revealed that left ventricular ejection fraction was higher in women than in men, in agreement with previous reports.

Future plans A gender questionnaire assessing sociocultural aspects implemented as part of the follow-up described here will allow to calculate a gender score and its evaluation based on the newly collected data. At the same time, the other BASE-II research foci established over the past 10 years will be continued and strengthened by the BASE-II transition into a longitudinal study with follow-up data on the older subsample.

Trial registration number DRKS00016157.

\section{INTRODUCTION}

The original BASE-II cohort

The Berlin Aging Study II (BASE-II) was launched as a multidisciplinary study aimed at better understanding the multitude of different ways in which age and ageing
Strengths and limitations of this study

- The GendAge study focuses on major risk factors for cardiovascular and metabolic diseases and on the development of major outcomes from intermediate phenotypes in the context of sex and gender differences.

- The Berlin Aging Study II (BASE-II) follow-up as part of the GendAge study assessments covered most of the medical, psychosocial and cognitive domains and variables assessed at baseline.

- Comprehensive and longitudinal study data offer the potential to answer a number of questions that are of crucial relevance for the health of old women and men.

- The possibility of a selection bias in the follow-up study population is a limitation, which we have made various efforts to accommodate.

- We are able to systematically quantify the sampling bias and even account for it when it comes to the question of generalisability of study results to a population as a whole.

evolve and identifying the underlying mechanisms and contributing factors. Baseline recruitment of 2200 adult volunteers from the Berlin metropolitan area and baseline assessments were completed in 2014. ${ }^{1}$ The ascertainment protocol included the collection of data from different domains for each of the 2200 participants (about $75 \%$ aged 60 years and above, the older group of BASE-II participants), namely, geriatrics and internal medicine, immunology, genetics, psychology, sociology and economics. ${ }^{12}$

BASE-II baseline data were used in a multitude of analysis projects focusing on key questions revolving around age and ageing. Research topics of the ongoing study include, but are not limited to, cognitive ageing, ${ }^{3-5}$ cardiovascular and metabolic health, ${ }^{6-8}$ sarcopenia and frailty, ${ }^{9}{ }^{10}$ psychosocial factors of 
ageing, ${ }^{11} 12$ genetic risk factors of ageing and disease, ${ }^{13-15}$ the impact of characteristics of the neighbourhood people are living in, ${ }^{16}$ as well as indicators of biological age $^{1718}$ and immune biomarkers. ${ }^{19}$ For an overview of the BASE-II research foci and publications, refer to a previous work $^{20}$ and the BASE-II website (https://www.base2.mpg. de/en/project-information/publications).

\section{Contact procedure: follow-up assessments}

Of the original BASE-II sample consisting of 2200 participants, 1671 aged 60 years and above (=older group) were assessed medically at baseline between 2009 and 2014 . The follow-up assessments within the GendAge study took place between 22 June 2018 and 10 March 2020 at the Charité Universitaetsmedizin Berlin. During the recruitment of the follow-up cohort, we approached all BASE-II participants of the remaining pool of 1428 subjects out of the originally 1671 subjects who completed the baseline medical assessments at an age of 60 years and older (older BASE-II group, see figure 1). Between 7 February 2020 and 13 March 2020, we additionally performed follow-up assessments in a total of 64 participants of the younger BASE-II group aged 20-35 years at baseline until these assessments were suspended because of the SARS-CoV-2 pandemic. Potential follow-up participants were contacted via telephone and an invitation letter that contained a comprehensive participant's information sheet. Letters of consent were sent at least 5 days before the scheduled first of two assessment days to all subjects who agreed to participate. As a result of a 4-week pilot phase, we reduced the maximum number of participants examined on each of the first two study days from 6 to 4 , with an interval of usually 7 days between study visit 1 and 2. Largely because of this early adjustment, follow-up examinations lasted 21 months instead of the 15 months originally planned. Moreover, another wave of cognitive assessments carried out by the Max Planck Institute for Human Development (MPIB) has been tightly linked to the GendAge assessment of BASE-II participants. The cognitive session (=third study visit) followed about 7 days after the second medical examination.

\section{What is the reason for the new data collection?}

The study 'Sex- and gender-sensitive prevention of cardiovascular and metabolic disease in older adults in Germany', the GendAge study, focuses on major risk factors for cardiovascular and metabolic diseases and on the development of major outcomes from intermediate phenotypes in the context of biological sex and gender differences. Major outcomes include, but are not limited to, myocardial infarction (MI), heart failure and diabetes mellitus type 2 (T2D), as well as mortality and quality of life. Gender was quantitated in two ways: by a retrospective approach, based on available data at study entry (20092014) and already published (GenderScore-I, GS-I) ${ }^{21}$ as well as by a comprehensive gender questionnaire covering a range of sociocultural gender characteristics as a central instrument (GenderScore-II, GS-II). This questionnaire contains an adapted version of the gender questionnaire developed by Pelletier and colleagues covering most of the four gender aspects described by the Women Health Research Network of the Canadian Institute of Health Research (gender roles, gender identity, gender relations and institutionalised gender). ${ }^{22} 23$ The variables finally constituting the GS-I were chronic stress, marital status, risk-taking behaviour, personality attributes: agreeableness, neuroticism, extraversion, loneliness, conscientiousness and level of education. ${ }^{21}$

\section{What will be the new areas of research?}

There is new knowledge showing that sex differences play a role in all major diseases, their prevention and treatment. ${ }^{24}$ Other studies showed that gender as the sociocultural dimension of being a woman or a man affects disease and treatment outcomes and also well-being. ${ }^{22} 25$ The new areas of research cover the systemic inclusion of sex-specific analysis and the inclusion of gender. Ageing interacts with sex and gender differences in health, but it is not clear, which mechanisms are most important.

GendAge aims to better understand, which mechanisms affect cardiometabolic morbidity, mortality and quality of life among older adults in a sex-sensitive and gender-sensitive manner.

While on different occasions follow-up data were ascertained for questionnaire and cognitive data, ${ }^{56-30}$ as being part of the GendAge study, this cohort profile update describes the first comprehensive follow-up assessments in a BASE-II subsample (older group) that also includes

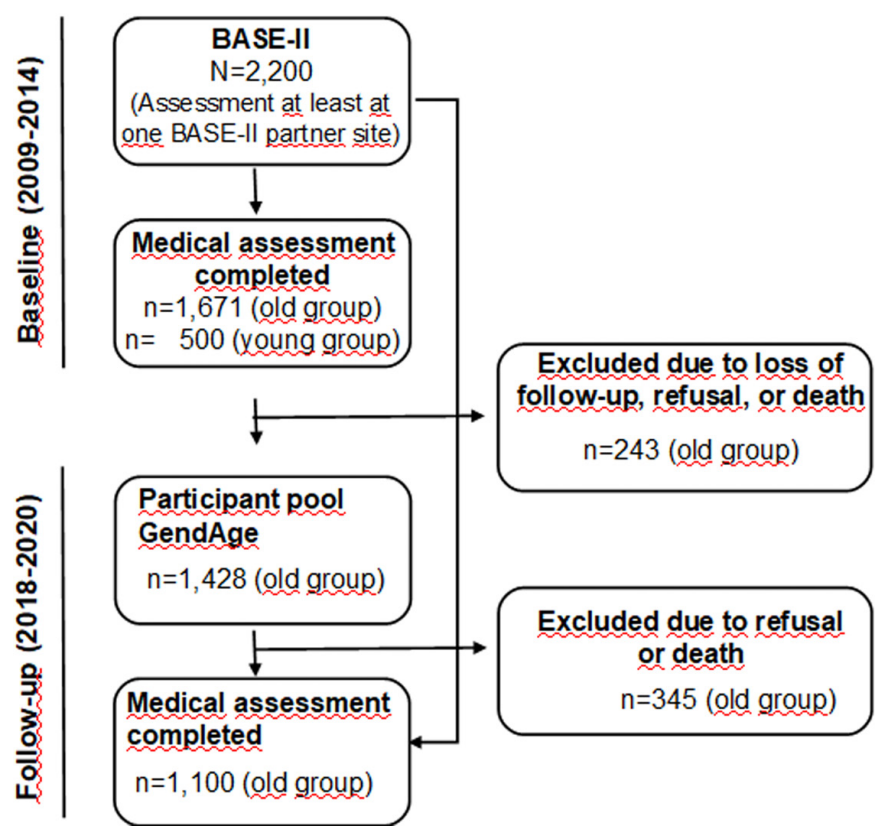

Figure 1 Flowchart explaining the final Berlin Aging Study II (BASE-II) sample with follow-up assessments completed in GendAge. A total of 17 of the 1,100 participants examined atfollow-up were not medically examined at baseline but were examined at least atone of the other BASE-II study sites. 
a reassessment of central variables in the areas of internal medicine and geriatrics.

\section{COHORT DESCRIPTION}

As presented in the flowchart (figure 1), following the contact procedure until the participant pool was exhausted resulted in a total of 1100 participants of the older BASE-II group investigated in the follow-up. These participants had a mean age of 75.6 years ( $\mathrm{SD} \pm 3.8$, range 64.9-94.1 years), with up to 10.4 years of follow-up (mean follow-up at 7.4 years, $\mathrm{SD} \pm 1.5$ ). At follow-up, almost all of the older participants were retired (97.3\%) as compared with $86 \%$ at the time of baseline assessment. At baseline, BASE-II participants were characterised by higher education and better self-reported health status than the general population of Berlin and Germany. ${ }^{1}$ At follow-up, this selection seems to have increased, with $68.8 \%$ of the participants reported to have a high school degree (51\% at baseline) and about $61 \%$ rated their health as very good or $\operatorname{good}(40 \%$ at baseline). The rate of divorce had been above average at baseline with $29 \%$ and had dropped to $21.7 \%$ at follow-up, which is still significantly above the German and Berlin average (ie, $12.0 \%$ and $17.4 \%$, respectively), ${ }^{31}$ while the proportion of widowed participants increased from $5 \%$ at baseline to $10.5 \%$ in the follow-up dataset of older BASE-II participants. As shown in table 1, differences between men and women are evident with respect to the sociodemographic status and psychosocial functioning in the follow-up cohort: Men reported significantly higher school degrees and higher satisfaction with life in general than women. Interestingly, self-rated health did not differ between men and women, which matches to the overall morbidity estimated by an adapted version of the Charlson morbidity index, ${ }^{17} 32$ which also did not differ between men and woman $(\mathrm{p}=0.98$, table 1$)$. This morbidity index, however, increased between baseline and follow-up $(\mathrm{p}<0.001$, Wilcoxon signed-rank test and data not shown). Differences between men and women exist in the follow-up dataset with respect to the prevalence of some, but not all cardiovascular risk factors and diseases (table 1). Men, for example, had a higher BMI and a higher proportion of men reported to have T2D and MI than women. No significant differences between men and women were evident in the reporting of hypertension, peripheral artery disease and stroke. With the aim of investigating human ageing processes in BASE-II under consideration of different disciplines and longitudinally, the baseline investigation aimed at the most comprehensive data collection possible. At follow-up, most of these data in the field of geriatrics, internal medicine and psychology were again part of the study protocol (for a select overview, see table 2).

\section{Findings to date}

With a focus on cardiometabolic diseases in GendAge, we extended the broad range of data assessed in this area at baseline by echocardiography. Data on right and left ventricular and atrial morphology and systolic and diastolic function and vascular stiffness were obtained. Left ventricular ejection fraction was higher in women than in men, in agreement with previous reports. ${ }^{33} 34$ Furthermore, increased LV mass and volumes in men before and after indexing to body surface area were confirmed, underscoring major sex differences in cardiovascular pathophysiology. ${ }^{35}$

With the aim of achieving a particularly high-quality standard in the assessment of participant's medical history at baseline and follow-up, including past and current diseases, the information given by the participants was recorded from study physicians as part of a structured one-to-one interview, allowing to consider its plausibility. This, however, does not cover the gap between reported (anamnestic) diseases and the diseases diagnosed in the course of the study. This is exemplified by T2D, which was reported by $15.6 \%$ and $8.6 \%$ of men and women, respectively. In contrast, this disease was diagnosed in $20.7 \%$ of men and $13.3 \%$ of women based on the American Diabetes Association guidelines 2019, ${ }^{36}$ indicating that a substantial proportion of almost $30 \%$ was unaware of the disease (table 1).

As part of our endeavours, we have developed a retrospective gender score taking BASE-II baseline data reflecting sociocultural aspects (eg, level of education, marital status and chronic stress) into account. This retrospective gender score (GS-I) was associated with a number of clinical and psychosocial variables and performed better in predicting differences in a subset of variables (eg, depression and life satisfaction) compared with biological sex. ${ }^{21}$ In addition, we have implemented a comprehensive gender questionnaire as part of the follow-up assessments described here, to calculate a prospective gender score as proposed by Pelletier and colleagues. ${ }^{22}$

Peripheral blood mononuclear cells were prepared from 903 participants at follow-up, of which 845 were fully analysable (58 were dropouts) and frequencies as well as absolute counts of recent thymic emigrants (RTEs), $T_{\text {EMRA }}$ effector $\mathrm{T}$ cell subsets $\left(\mathrm{T}_{\text {EMRA }}\right)$ and cytotoxic $\mathrm{CD} 4^{+} \mathrm{T}$ cells were directly assessed. While RTEs are known to decrease with ageing, ${ }^{37}$ alterations in $\mathrm{T}_{\text {EMRA }}$ and specialised cytotoxic $\mathrm{CD}^{+} \mathrm{T}$ cell compartments can be indicative of agerelated perturbations of systemic $\mathrm{T}$ cell immunity. ${ }^{38}$ The immunological screening has so far revealed significantly higher frequencies of RTEs in women as compared with men, indicating a higher thymic $\mathrm{T}$ cell production even at the advanced ages of the GendAge participants. In men, more $\mathrm{CD}_{45 \mathrm{RA}^{+}}$re-expressing $\mathrm{T}_{\mathrm{EMRAs}}$ were detected than in women (table 1). These cells are associated with chronic viral infections (eg, CMV) and can serve as a signature of immune-senescence. ${ }^{39}$ We found no significant difference in the frequencies of cytotoxic $\mathrm{CD} 4^{+}$ $\mathrm{T}$ cells. Together, these preliminary findings confirm the better immune status of aged women as compared with men. A detailed analysis of the datasets will identify additional correlates of sex and gender, ageing and the immune system. 
Table 1 Selection of BASE-II follow-up characteristics as assessed of theGendAge study

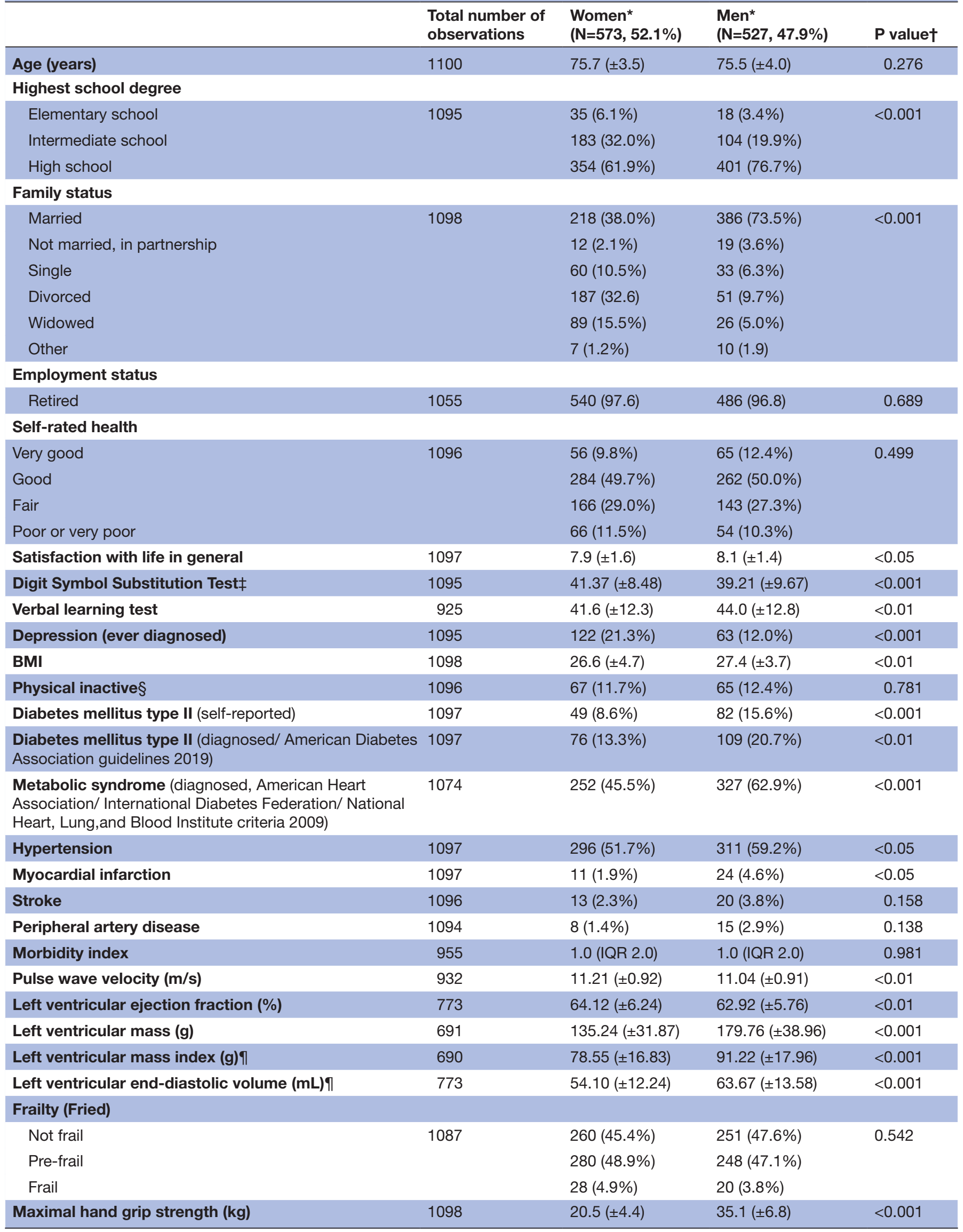




\begin{tabular}{|c|c|c|c|c|}
\hline & $\begin{array}{l}\text { Total number of } \\
\text { observations }\end{array}$ & $\begin{array}{l}\text { Women* } \\
(\mathrm{N}=573,52.1 \%)\end{array}$ & $\begin{array}{l}\text { Men* } \\
(\mathrm{N}=527,47.9 \%)\end{array}$ & P value \\
\hline Recent thymic emigrants (naïve $\mathrm{CD} 4^{+} \mathrm{T}$ cells) & $395^{\star \star}$ & $64.69( \pm 16.34)$ & $51.03( \pm 13.69)$ & $<0.001$ \\
\hline Cytotoxic SLAMF7+CD4 ${ }^{+} \mathrm{T}$ cells & $181 \dagger \dagger$ & $6.02( \pm 5.98)$ & $6.05( \pm 6.60)$ & 0.974 \\
\hline
\end{tabular}

${ }^{*}$ Data are presented as $\mathrm{N}(\%)$, mean \pm SD or median (IQR).

†Differences between women and men were assessed using the parametric t-test, the non-parametric Mann-Whitney $U$ test or the $\chi^{2}$ where appropriate.

$\ddagger$ Assessed at study visit 1 .

$\S$ Assessed with the question, 'Are you seldom or never physically active?'.

ๆAdjusted for body surface area.

${ }^{\star \star} 845$ expected to be available after completion of the analyses.

††629 expected to be available after completion of the analyses.

$\mathrm{BMI}$, body mass index.

The gender questionnaire implemented as part of the follow-up assessments described here will allow to calculate a gender score and its evaluation based on the newly collected clinical and psychosocial follow-up data. At the same time, the other BASE-II research foci established over the past 10 years will be continued and strengthened with the transition of BASE-II into a longitudinal study with follow-up data on the older subsample.

\section{Other measurements}

Similar to baseline, we determined numerous routine laboratory parameters from blood and urine (table 2), and also stored blood plasma/serum and urine samples for future analyses. Genomic DNA was already extracted from EDTA-blood and buccal swab samples from GendAge participants, which will be used, for example, for the profiling of genome-wide DNA methylation signatures and new genome-wide single nucleotide polymorphism genotyping experiments (table 2). In between the two assessment days at the Charite, participants were asked to fill out a comprehensive psychosocial take-home questionnaire and return this at their second Charité visit.

At baseline, the BASE-II included a group of 600 younger subjects aged 20-35 years serving as a reference population, ${ }^{1}$ of which 500 completed baseline medical assessments. Between 7 February 2020 and 13 March 2020, we performed follow-up assessments in a total of 64 participants of this younger group until these assessments were suspended because of the SARS-CoV-2 pandemic. These younger participants had a mean age of 36.8 years ( $\mathrm{SD} \pm 3.5$, range $29.3-44.1$ years), with up to 10.7 years of follow-up (minimum 6.1 years, mean follow-up at 8.2 years, SD \pm 1.6 ). Follow-up for these younger BASE-II participants essentially followed the protocol used for the 1100 older BASE-II participants. Because this younger group is not primarily part of the analyses planned in GendAge, further details about this group will be described elsewhere.

The cognitive session carried out by the MPIB lasted about 4.5-5 hours (third study visit). Subjects were tested in groups of 4-6 individuals. The cognitive battery included 17 measures of learning and memory performance, attention/processing speed, working memory, executive functioning and perceptual speed (see table 2). Within the week between study visit 2 (Charité) and 3 (MPIB), accelerometers (ActiGraph wGT3X-BT) have been used to track participants' physical activity and sleep in a subset of our participants $(n=750)$.

After the cognitive session, participants were invited to take part in a one-to-one interview on a different day. This additional individual assessment took up to $60 \mathrm{~min}$ and serves as a cohort comparison between the BASE and BASE-II study populations. This additional data collection will also contribute to the BASE-II cognitive waves, allowing us to further investigate individual differences in ageing trajectories (for an overview, refer to previous work $^{20}$ ).

Furthermore, and as part of a collaboration with the Lifebrain study, a consortium of European studies funded by the EU Horizon 2020 Framework Programme, ${ }^{40}$ we collected blood samples using dried blood cards, in order to determine laboratory parameters with identical methods used for all Lifebrain participating sites. Lifebrain aims at identifying determinants of healthy lifespan development by integrating and harmonising data and results from 11 large and predominantly longitudinal European samples from seven countries. This has yielded a database of fine-grained measures focusing on brain and cognition from more than 7000 individual participants.

The GendAge study was approved by the Ethics Committee of the Charité-Universitätsmedizin Berlin (approval number EA2/144/16) and all participants gave written informed consent. GendAge is registered in the German Clinical Trials Register (Study-ID: DRKS00016157). The cognitive battery was approved by the Ethics Committee of the Max-Planck-Institute and the genomics experiments were approved by the Ethics Committees of the Charité (approval number EA2/144/16) and the University of Lübeck (approval numbers AZ19-390A and 19-391A). 
Table 2 BASE-II follow-up assessments during the two GendAge study visits and the cognitive sessions (third study visit)

\section{Type of assessment/domain Example assessments/tests}

Physical examination and medical Medical history structured by organ systems, medication, body weight, height, lifestyle (including history smoking status, alcohol consumption, physical activity)

Physical status and functional tests

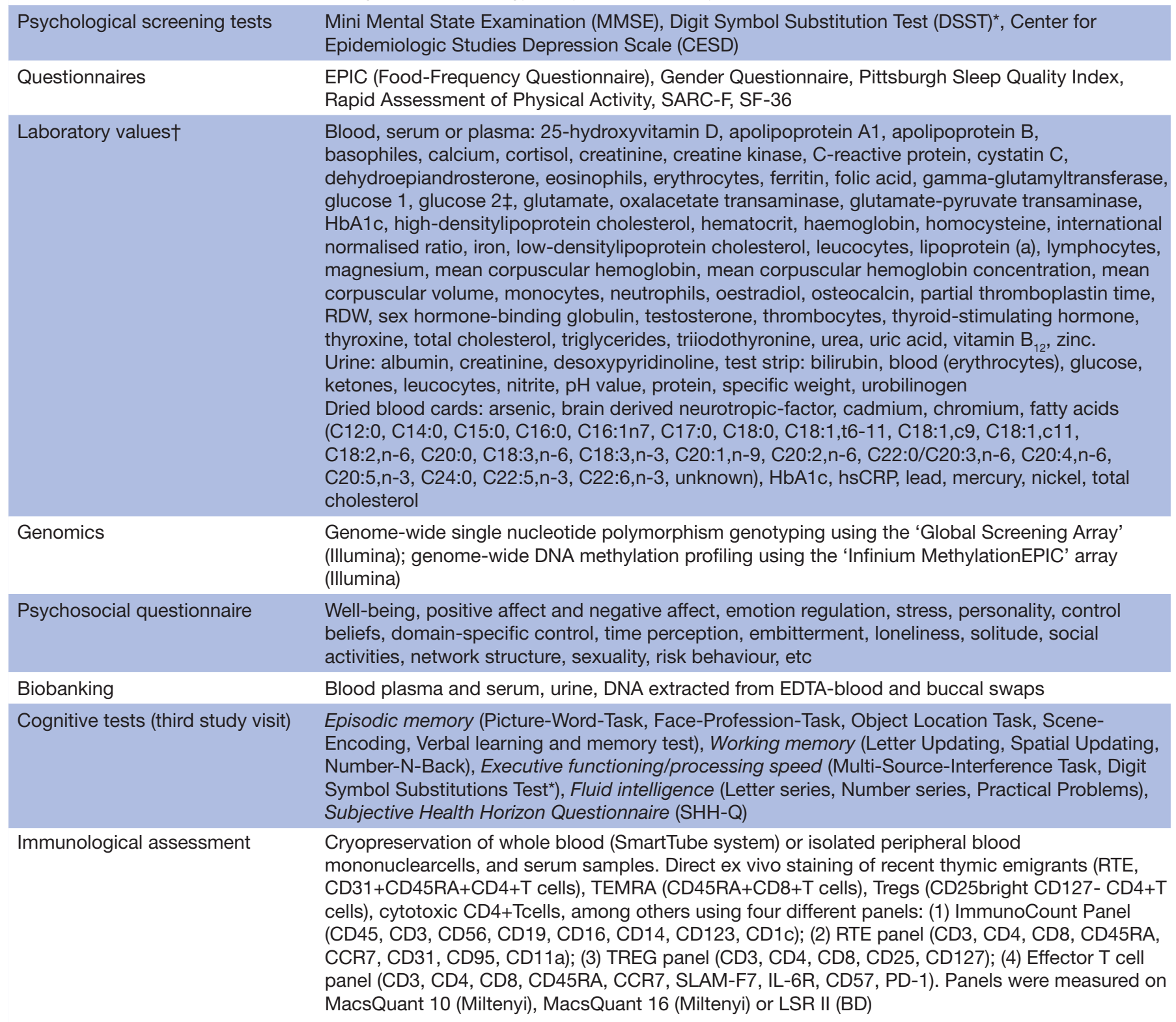

${ }^{*}$ Assessed at study visit 1 and visit 3 .

†Blood samples were drawn after a fasting period of at least 8 hours (if not otherwise indicated).

†Post-load (75 g glucose, 2 hours), not assessed in participants with known diabetes.

BASE-II, Berlin Aging Study II.

\section{Strengths and limitations}

The BASE-II follow-up assessments covered most of the medical, psychosocial and cognitive domains, and variables assessed at baseline, and thereby taking the BASE-II characteristic of an exceptionally broad and in-depth data collection to a next, longitudinal level. In addition, and in the context of the GendAge focus on cardiometabolic disease, we extended the assessments in this area, for example, by including high-quality echocardiography resulting in a unique data collection. This strength with respect to comprehensive and longitudinal data offers the potential to answer a number of questions that are of 
crucial relevance for the health of old women and men. Thus, GendAge will make important contributions for improvements in understanding the health and well-being of older adults in both genders. BASE-II was initiated as a multidisciplinary study with expertise in a broad range of fields relevant for ageing research (eg, internal medicine and geriatrics, biology, psychology, genetics, immunology, socioeconomics and now in GendAge further extended by sociocultural aspects of gender). The past 10 years of BASE-II research have shown that multidisciplinary collaboration is not only a statement of intent, but a fruitbearing working posture and a clear strength of BASE-II.

Sampling bias is a challenge which cohort studies have to deal with, and this is especially an issue in the follow-up of older study populations such as the older group of BASE-II participants. To address this, we have made a considerable effort (eg, offering a taxi service for participants not able to travel independently) to include as many participants in the follow-up as possible. Additionally, and similar to baseline, we are able to systematically quantify the sampling bias and even account for it when it comes to the question of generalisability of study results to a population as a whole (eg, Berlin or Germany), due to the evaluation of selectivity and representativeness via the German Socio-Economic Panel Study (SOEP). ${ }^{1}$ Despite these possibilities, we cannot rule out the possibility of a selection bias completely, which certainly is a weakness of this study, a weakness that applies to all cohort studies relying on voluntary participants who have been nonrandomly recruited. With our direct comparability to the national representative SOEP study, we are in a position though to quantify the amount of selectivity and, if need, take measures to correct and adjust our results.

\section{Author affiliations}

${ }^{1}$ Department of Endocrinology and Metabolic Diseases (including Division of Lipid Metabolism), Charité - Universitätsmedizin Berlin, corporate member of Freie Universität Berlin and Humboldt-Universität zu Berlin, and Berlin Institute of Health at Charité - Universitätsmedizin Berlin, Berlin, Germany

${ }^{2} \mathrm{BCRT}$ - Berlin Institute of Health Center for Regenerative Therapies, Berlin Institute of Health at Charité - Universitätsmedizin Berlin, Berlin, Germany

${ }^{3}$ Department of Psychology, Humboldt University of Berlin, Berlin, Berlin, Germany

${ }^{4}$ Center for Lifespan Psychology, Max-Planck-Institute for Human Development, Berlin, Germany

${ }^{5}$ Charité - Universitätsmedizin Berlin, corporate member of Freie Universität Berlin, Humboldt-Universität zu Berlin, and Berlin Institute of Health; Institute of Social Medicine, Epidemiology and Health Economics, Berlin, Germany

${ }^{6}$ DZHK (German Centre for Cardiovascular Research), Partner site Berlin, Berlin, Germany

${ }^{7}$ Berlin Institute for Gender in Medicine, Charite Universitatsmedizin Berlin, Berlin, Germany

${ }^{8} \mathrm{Si}-\mathrm{M}$ / "Der Simulierte Mensch" a science framework of Technische, Universitat Berlin andCharité - Universitatsmedizin Berlin, Berlin, Germany

${ }^{9}$ Regenerative Immunology and Aging, BIH Center for Regenerative Therapies, Charité Universitatsmedizin Berlin, Berlin, Germany

${ }^{10}$ Lübeck Interdisciplinary Platform for Genome Analytics, University of Lübeck, Lübeck, Germany

${ }^{11}$ Center for Lifespan Changes in Brain and Cognition (LCBC), Dept of Psychology, University of Oslo, Oslo, Norway

${ }^{12}$ Max Planck UCL Centre for Computational Psychiatry and Ageing Research, Berlin, Germany
${ }^{13}$ Department of Cardiology, University Hospital Zürich, University of Zürich, Zürich, Switzerland

Acknowledgements We are deeply indebted to all individuals of the BASE-II cohort who have agreed to participate in the follow-up assessments. We would further like to thank all staff members and students involved in the medical examinations of participants and documentation, especially Nora Berger, Janina Dombrowski, Mergim Domuzeti, Elisa Dreißig, Ilona Enarovic, Anthony Ganswindt, Thomas Grenkowitz, Anna Hunold, llias Katsianas, Yoo-Ri Kim, Jörn Kiselev, Paula Krull, Christine Kytmannow, Elisa Lemke, Julia Mätzkow, Charlotte Mentzel, Nadja Mielke, Narantuya Mishig, Angela Motz, Eduard Nitschke, Aabi Okute, Danai Pantelakis, Sophie Poser, Johanne Spieker and Taleka Vollmar (all Charité Universitaetsmedizin Berlin, Biology of Aging group); and Luisa Lüth, Mariebelle Kaus, Isabel Ganter, Marlene Rosendahl, Jasmin Boneberger, Antonia Sprenger, Hania El-Kersh (all Humboldt University, Department of Psychology), and Kirsten Becker for the excellent daily organization of the running study within and between the research units, the Telefonstudio for their daily contact with the participants, Martin Becker for the continuous quality checks of our sample and Berndt Wischnewski for updating the database and cognitive battery (all Max Planck Institute for Human Development).We kindly acknowledge the excellent cooperation with the Central Biomaterial Bank, the joint core facility of the CharitéUniversitätsmedizin Berlin and the Berlin Institute for Health (BIH). Furthermore, we appreciate the great support by the BIH-REDCap team and especially from Andreas Hetey. We would like to acknowledge the assistance of the CharitélBIH Cytometry Core.

Collaborators Additional BASE-II/GendAge investigators: Nikolaus Buchmann Peter Eibich ${ }^{\text {ii, }}$, Friederike Kendel ${ }^{\text {iii, }}$,Maximilian König ${ }^{\text {iv }}$, Christina M. Lill ${ }^{v, v i}$, Maike Mangold $^{\text {vii }}$, Ahmad Tauseef Nauman ${ }^{\text {iii }}$, Kristina Norman ${ }^{\text {viii, ix }}$, Graham Pawelec ${ }^{x, x i}$, Sarah Toepfer ${ }^{\text {iv }}$, Valentin Max Vetteriv, Gert G. Wagnerii, Ursula Wilkenshoff', Kilian Wistuba-Hamprecht ${ }^{\text {xiiii }}$ Charité; Department of Cardiology, Charité - University Medicine Berlin (Campus Benjamin Franklin), Berlin, Germany; "Max Planck Institute for Demographic Research, Rostock, Germany; iii,Berlin Institute for Gender in

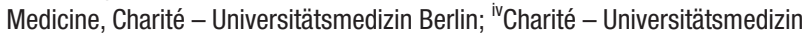
Berlin, corporate member of Freie Universität Berlin, Humboldt-Universität zu Berlin and Berlin Institute of Health; Department of Endocrinology and Metabolism, Berlin, Germany; ' Section for Translational Surgical Oncology and Biobanking, Department of Surgery, University of Lübeck and University Medical Center Schleswig-Holstein, Campus Lübeck, 23552 Lübeck, Germany; vi Ageing Epidemiology Research Unit, School of Public Health, Imperial College, London SW71, UK; vii Regenerative Immunology and Aging, BIH Center for Regenerative Therapies, Charité

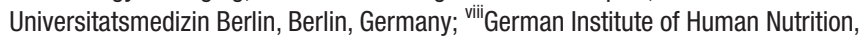
Department of Nutrition and Gerontology, Potsdam-Rehbruecke (DlfE), Germany; ${ }^{i x}$ Charité - Universitätsmedizin Berlin, Forschungsgruppe Geriatrie am EGZB, Berlin, Berlin, Germany; 'Department of Immunology, University of Tübingen, Tübingen, Germany; ${ }^{x}$ Health Sciences North Research Institute, Sudbury, ON, Canada;

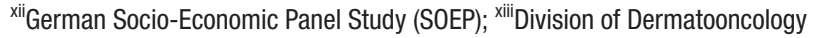
Department of Dermatology, University of Tübingen, Tübingen, Germany.

Contributors Conceived and designed the study: ID, VR-Z, SD, UL and DG. Collected study specific data: ID, VB, JD, SD, US, DS, ET, JB, LB and AT. Providing BASE-II baseline data: DG, ES-T, ID, JD, LB, SD and UL. Analysed the data: ID, ET and JB. Wrote the manuscript: ID. All authors revised and approved the manuscript.

Funding The GendAge study research project (Co-PIs are Ilja Demuth, Vera Regitz-Zagrosek, and Denis Gerstorf) is supported by the German Federal Ministry of Education and Research (Bundesministerium für Bildung und Forschung, BMBF) grant numbers 01GL1716A (ID and VRZ) and 01GL1716B (DG). Genomics assessments are funded by the Cure Alzheimer's Fund (as part of the "CIRCUITS-AD" consortium project) and the European Research Council's "Horizon2020" funding scheme (as part of the "Lifebrain" consortium project; both to LB). Additional contributions (e.g., equipment, logistics, personnel) are made from each of the participating sites.

\section{Competing interests None declared.}

Patient and public involvement Patients and/or the public were not involved in the design, or conduct, or reporting or dissemination plans of this research.

Patient consent for publication Not required.

Provenance and peer review Not commissioned; externally peer reviewed.

Data availability statement Data are available on reasonable request. The GendAge study principal investigators welcome new collaborations with other investigators. Interested investigators are invited to contact the study coordinating 
PI Ilja Demuth at ilja.demuth@charite.de to obtain additional information about the GendAge study and the data-sharing application form.

Open access This is an open access article distributed in accordance with the Creative Commons Attribution Non Commercial (CC BY-NC 4.0) license, which permits others to distribute, remix, adapt, build upon this work non-commercially, and license their derivative works on different terms, provided the original work is properly cited, appropriate credit is given, any changes made indicated, and the use is non-commercial. See: http://creativecommons.org/licenses/by-nc/4.0/.

ORCID iD

Ilja Demuth http://orcid.org/0000-0002-4340-2523

\section{REFERENCES}

1 Bertram L, Böckenhoff A, Demuth I, et al. Cohort profile: the Berlin aging study II (BASE-II). Int J Epidemiol 2014;43:703-12.

2 Gerstorf D, Bertram L, Lindenberger U, et al. Editorial. Gerontology 2016;62:311-5.

3 Hensel C, Becker M, Düzel S, et al. Influence of nutritional tyrosine on cognition and functional connectivity in healthy old humans. Neuroimage 2019;193:139-45.

4 Bender AR, Brandmaier AM, Düzel S, et al. Hippocampal subfields and limbic white matter jointly predict learning rate in older adults. Cereb Cortex 2020;30:2465-77.

5 Dahl MJ, Mather M, Düzel S, et al. Rostral locus coeruleus integrity is associated with better memory performance in older adults. Nat Hum Behav 2019;3:1203-14.

6 Buchmann N, Scholz M, Lill CM, et al. Association between lipoprotein(a) level and type 2 diabetes: no evidence for a causal role of lipoprotein(a) and insulin. Acta Diabetol 2017;54:1031-8.

7 Düzel S, Buchmann N, Drewelies J, et al. Validation of a single factor representing the indicators of metabolic syndrome as a continuous measure of metabolic load and its association with health and cognitive function. PLoS One 2018;13:e0208231.

8 Röhr F, Bucholtz N, Toepfer S, et al. Relationship between Lipoprotein (a) and cognitive function - Results from the Berlin Aging Study II. Sci Rep 2020;10:10636.

9 Spira D, Buchmann N, König M, et al. Sex-Specific differences in the association of vitamin $\mathrm{D}$ with low lean mass and frailty: results from the Berlin aging study II. Nutrition 2019;62:1-6.

10 Spira D, Buchmann N, Nikolov J, et al. Association of low lean mass with frailty and physical performance: a comparison between two operational definitions of Sarcopenia-Data from the Berlin aging study II (BASE-II). J Gerontol A Biol Sci Med Sci 2015;70:779-84.

11 Gerstorf $D$, Hülür G, Drewelies J, et al. Secular changes in late-life cognition and well-being: towards a long bright future with a short brisk ending? Psychol Aging 2015;30:301-10.

12 Hülür G, Drewelies J, Eibich P, et al. Cohort differences in psychosocial function over 20 years: current older adults feel less Lonely and less dependent on external circumstances. Gerontology 2016;62:354-61.

13 Lu Y, Day FR, Gustafsson S, et al. New loci for body fat percentage reveal link between adiposity and cardiometabolic disease risk. Nat Commun 2016;7:10495.

14 Nikpay M, Goel A, Won H-H, et al. A comprehensive 1,000 Genomesbased genome-wide association meta-analysis of coronary artery disease. Nat Genet 2015;47:1121-30.

15 Schmidt AF, Swerdlow DI, Holmes MV, et al. Pcsk9 genetic variants and risk of type 2 diabetes: a Mendelian randomisation study. Lancet Diabetes Endocrinol 2017;5:97-105.

16 Eibich P, Krekel C, Demuth I, et al. Associations between neighborhood characteristics, well-being and health vary over the life course. Gerontology 2016;62:362-70.

17 Meyer A, Salewsky B, Spira D, et al. Leukocyte telomere length is related to appendicular lean mass: cross-sectional data from the Berlin aging study II (BASE-II). Am J Clin Nutr 2016;103:178-83.

18 Vetter VM, Spira D, Banszerus VL, et al. Epigenetic Clock and Leukocyte Telomere Length are Associated with Vitamin D Status, but not with Functional Assessments and Frailty in the Berlin Aging Study II. J Gerontol A Biol Sci Med Sci 2020.

19 Wistuba-Hamprecht K, Haehnel K, Janssen N, et al. Peripheral blood T-cell signatures from high-resolution immune phenotyping of $\gamma \delta$ and $\alpha \beta$ T-cells in younger and older subjects in the Berlin aging study II. Immun Ageing 2015;12:25.

20 Demuth let al. Berlin aging study II (BASE-II). Encyclopedia of Gerontology and Population Aging 2019:1-8.

21 Nauman AT, Behlouli H, Alexander N, et al. Gender score development in the Berlin aging study II: a retrospective approach. Biol Sex Differ 2021;12:15.

22 Pelletier R, Ditto B, Pilote L. A composite measure of gender and its association with risk factors in patients with premature acute coronary syndrome. Psychosom Med 2015;77:517-26.

23 Johnson JL, Greaves L, Repta R. Better science with sex and gender: a primer for health research. Vancouver: Women's Health Research Network, 2007.

24 Mauvais-Jarvis F, Bairey Merz N, Barnes PJ, et al. Sex and gender: modifiers of health, disease, and medicine. Lancet 2020;396:565-82.

25 Lacasse A, Pagé MG, Choinière M, et al. Conducting gender-based analysis of existing databases when self-reported gender data are unavailable: the gender index in a working population. Can J Public Health 2020;111:155-68.

26 Gerstorf D, Drewelies J, Duezel S, et al. Cohort differences in adultlife trajectories of internal and external control beliefs: a tale of more and better maintained internal control and fewer external constraints. Psychol Aging 2019;34:1090-108.

27 Potter S, Drewelies J, Wagner J, et al. Trajectories of multiple subjective well-being facets across old age: the role of health and personality. Psychol Aging 2020;35:894-909.

28 Graham EK, Weston SJ, Gerstorf D, et al. Trajectories of big five personality traits: a coordinated analysis of 16 longitudinal samples. Eur J Pers 2020;34:301-21.

29 Mantantzis K, Drewelies J, Duezel S, et al. Dehydration predicts longitudinal decline in cognitive functioning and well-being among older adults. Psychol Aging 2020;35:517-28.

30 Mueller S, Wagner J, Drewelies J, et al. Personality development in old age relates to physical health and cognitive performance: evidence from the Berlin aging study II. J Res Pers 2016;65:94-108.

31 Goebel J, Grabka MM, Liebig S, et al. The German socio-economic panel (SOEP). Jahrbücher für Nationalökonomie und Statistik 2019;239:345-60.

32 Charlson ME, Pompei P, Ales KL, et al. A new method of classifying prognostic comorbidity in longitudinal studies: development and validation. J Chronic Dis 1987:40:373-83.

33 Chung AK, Das SR, Leonard D, et al. Women have higher left ventricular ejection fractions than men independent of differences in left ventricular volume: the Dallas heart study. Circulation 2006;113:1597-604.

34 Asch FM, Miyoshi T, Addetia K, et al. Similarities and differences in left ventricular size and function among races and Nationalities: results of the world alliance societies of echocardiography normal values study. J Am Soc Echocardiogr 2019;32:1396-406.

35 Yeon SB, Salton CJ, Gona P, et al. Impact of age, sex, and indexation method on Mr left ventricular reference values in the Framingham heart study offspring cohort. J Magn Reson Imaging 2015;41:1038-45.

36 American Diabetes Association. 2. Classification and Diagnosis of Diabetes: Standards of Medical Care in Diabetes-2019. Diabetes Care 2019;42:S13-28.

37 Kohler S, Thiel A. Life after the thymus: CD31+ and CD31- human naive CD4+ T-cell subsets. Blood 2009;113:769-74.

38 Weyand CM, Brandes JC, Schmidt D, et al. Functional properties of CD4+ CD28- T cells in the aging immune system. Mech Ageing Dev 1998;102:131-47.

39 Di Benedetto S, Derhovanessian E, Steinhagen-Thiessen E, et al. Impact of age, sex and CMV-infection on peripheral T cell phenotypes: results from the Berlin BASE-II study. Biogerontology 2015;16:631-43.

40 Walhovd KB, Fjell AM, Westerhausen R, et al. Healthy minds from 0-100 years: Optimising the use of European brain imaging cohorts ("Lifebrain"). Eur Psychiatry 2018;47:76-7. 